\title{
Numerical Analysis of Fractional-Order Parabolic Equations via Elzaki Transform
}

\author{
Muhammad Naeem (D), ${ }^{1}$ Omar Fouad Azhar, ${ }^{1}$ Ahmed M. Zidan $\mathbb{D}^{2,3}$ \\ Kamsing Nonlaopon $\mathbb{D}^{4},{ }^{4}$ and Rasool Shah $\mathbb{D}^{5}$ \\ ${ }^{1}$ Deanship of Joint First Year Umm Al-Qura University Makkah, Saudi Arabia \\ ${ }^{2}$ Department of Mathematics, College of Science, King Khalid University, 9004 Abha, Saudi Arabia \\ ${ }^{3}$ Department of Mathematics, Faculty of Science, Al-Azhar University, 71524 Assiut, Egypt \\ ${ }^{4}$ Department of Mathematics, Faculty of Science, Khon Kaen University, Khon Kaen 40002, Thailand \\ ${ }^{5}$ Department of Mathematics, Abdul Wali Khan University, Mardan 23200, Pakistan
}

Correspondence should be addressed to Kamsing Nonlaopon; nkamsi@kku.ac.th and Rasool Shah; rasoolshah@awkum.edu.pk

Received 10 June 2021; Revised 1 August 2021; Accepted 11 August 2021; Published 1 September 2021

Academic Editor: Badr Saad T. Alkaltani

Copyright (c) 2021 Muhammad Naeem et al. This is an open access article distributed under the Creative Commons Attribution License, which permits unrestricted use, distribution, and reproduction in any medium, provided the original work is properly cited.

\begin{abstract}
This research article is dedicated to solving fractional-order parabolic equations, using an innovative analytical technique. The Adomian decomposition method is well supported by Elzaki transformation to establish closed-form solutions for targeted problems. The procedure is simple, attractive, and preferred over other methods because it provides a closed-form solution for the given problems. The solution graphs are plotted for both integer and fractional-order, which shows that the obtained results are in good contact with problems' exact solution. It is also observed that the solution of fractional-order problems is convergent to the integer-order problem. Moreover, the validity of the proposed method is analyzed by considering some numerical examples. The theory of the suggested approach is fully supported by the obtained results for the given problems. In conclusion, the present method is a straightforward and accurate analytical technique that can solve other fractional-order partial differential equations.
\end{abstract}

\section{Introduction}

The present research work is dedicated to studying the analytical solution of fractional-order parabolic equations. The literature is well recognized that a broad range of physics, engineering, nuclear physics, and mathematics problems can be defined as unique boundary and initial value problems. Homogeneous beam's transverse vibrations are controlled by fractional single fourth-order parabolic partial differential equations (PDEs). Such problem types occur in viscoelastic and inelastic flow mathematical modeling, layer deflection theories, and beam deformation [1-12]. Analyses of these problems have taken several physicist's and mathematician's attention [13-15].
The time fractional parabolic PDEs with variable coefficient:

$$
\begin{aligned}
& \frac{\partial^{\beta} \mu}{\partial \tau^{\beta}}+\kappa(\phi, \varphi, \psi) \frac{\partial^{4} \mu}{\partial \phi^{4}}+\frac{1}{\varphi} \mu(\phi, \varphi, \psi) \frac{\partial^{4} \mu}{\partial \varphi^{4}} \\
& +\frac{1}{\psi} \rho(\phi, \varphi, \psi) \frac{\partial^{4} \mu}{\partial \psi^{4}}=g(\phi, \varphi, \psi, \tau), \quad 1<\beta \leq 2, \tau \geq 0,
\end{aligned}
$$

where $\kappa(\phi, \varphi, \psi), \mu(\phi, \varphi, \psi)$, and $\rho(\phi, \varphi, \psi)$ are positive. With initial conditions, 


$$
\begin{gathered}
\mu(\phi, \varphi, \psi, \tau)=f_{0}(\phi, \varphi, \psi), \\
\mu_{\tau}(\phi, \varphi, \psi, \tau)=k_{0}(\phi, \varphi, \psi),
\end{gathered}
$$

with boundary conditions

$$
\begin{gathered}
\mu(a, \varphi, \psi, \tau)=h_{0}(\varphi, \psi, \tau), \mu(b, \varphi, \psi, \tau)=h_{1}(\varphi, \psi, \tau), \\
\mu(\phi, a, \psi, \tau)=g_{0}(\varphi, \psi, \tau), \mu(\phi, b, \psi, \tau)=g_{1}(\varphi, \psi, \tau), \\
\mu(\phi, \varphi, a, \tau)=k_{0}(\varphi, \psi, \tau), \mu(\phi, \varphi, b, \tau)=k_{1}(\varphi, \psi, \tau), \\
\mu_{\phi \phi}(a, \varphi, \psi, \tau)=\bar{h}_{0}(\varphi, \psi, \tau), \mu_{\phi \phi}(b, \varphi, \psi, \tau)=\overline{h_{1}}(\varphi, \psi, \tau), \\
\mu_{\varphi \varphi}(\phi, a, \psi, \tau)=\bar{g}_{0}(\varphi, \psi, \tau), \mu_{\varphi \varphi}(\phi, b, \psi, \tau)=\bar{g}_{1}(\varphi, \psi, \tau), \\
\mu_{\psi \psi}(\phi, \varphi, a, \tau)=\bar{k}_{0}(\varphi, \psi, \tau), \mu_{\psi \psi}(\phi, \varphi, b, \tau)=\overline{k_{1}}(\varphi, \psi, \tau) .
\end{gathered}
$$

For which, $h_{\ell}, g_{\ell}, k_{\ell}, h_{\ell}, g_{\ell}$, and $k_{\ell}$ are continuous variables, and $\ell$ differs between 0 and 1 and beam's flexural stiffness ratio [1] in its volume per unit mass, like, and its mentions $[1,3,4,6,8,10,11]$. Many researchers [10, 16, 17] have attempted to study the analytical solutions of the parabolic equation of the fourth-order. Different techniques have been suggested recently, such as the B-spline method [18], decomposition method [19], the implicit scheme [20], the explicit scheme [11], and the spline method [21] to analyze the solution of the partial differential fourth-order parabolic equation. Biazar and Ghazvini [22] have used He's iterative technique for the solution of parabolic PDE's. The modified version of this method was introduced in [23] to solve singular fourth-order parabolic PDEs. The fourth-order parabolic PDE analytical solution was examined in [24]. The modified Laplace discussed variational iteration technique [25] to solve singular fourth-order parabolic PDEs.

G. Adomian is an American scientist who has developed the Adomian decomposition method. It focuses on the search for a set of solutions and the decomposition of the nonlinear operator into a sequence in which Adomian polynomials [26] are recurrently computed to use the terms. This method is improved with the aid of Elzaki transformation such that the improved method is known as the Elzaki decomposition method (EDM). Elzaki Transform (ET) is a modern integral transform introduced by Tarig Elzaki in 2010. ET is a modified transform of Sumudu and Laplace transforms. It is important to note that there are many differential equations with variable coefficients that Sumudu and Laplace cannot accomplish transforms but can be conveniently done by using ET [27-30]. Many mathematicians have been solving differential equations with the aid of ET, such as Navier-Stokes equations [30], heat-like equations [31] and Burgers-Huxley equation [32].

\section{Preliminaries}

2.1. Definition. The Abel-Riemann of fractional operator $D^{\beta}$ of order $\beta$ is given as [27-30]
$D^{\beta} v(\zeta)=\left\{\begin{array}{l}\frac{d^{j}}{d \zeta^{j}} v(\zeta), \quad \beta=j, \\ \frac{1}{\Gamma(j-\beta)} \frac{d}{d \zeta^{j}} \int_{0}^{\zeta} \frac{v(\zeta)}{(\zeta-\psi)^{\beta-j+1}} d \psi, \quad j-1<\beta<j,\end{array}\right.$

where $j \in Z^{+}, \beta \in R^{+}$, and

$$
D^{-\beta} v(\zeta)=\frac{1}{\Gamma(\beta)} \int_{0}^{\zeta}(\zeta-\psi)^{\beta-1} v(\psi) d \psi, \quad 0<\beta \leq 1
$$

2.2. Definition. The fractional-order Abel-Riemann integration operator $J^{\beta}$ is defined as [27-30]

$$
J^{\beta} v(\zeta)=\frac{1}{\Gamma(\beta)} \int_{0}^{\zeta}(\zeta-\psi)^{\beta-1} v(\zeta) d \zeta, \quad \zeta>0, \beta>0 .
$$

The operator of basic properties:

$$
\begin{aligned}
J^{\beta} \zeta^{j} & =\frac{\Gamma(j+1)}{\Gamma(j+\beta+1)} \zeta^{j+\psi}, \\
D^{\beta} \zeta^{j} & =\frac{\Gamma(j+1)}{\Gamma(j-\beta+1)} \zeta^{j-\psi} .
\end{aligned}
$$

2.3. Definition. The Caputo fractional operator $D^{\beta}$ of $\beta$ is defined as [27-30]

$$
{ }^{C} D^{\beta} v(\zeta)=\left\{\begin{array}{l}
\frac{1}{\Gamma(j-\beta)} \int_{0}^{\zeta} \frac{\nu^{j}(\psi)}{(\zeta-\psi)^{\beta-j+1}} d \psi, \quad j-1<\beta<j, \\
\frac{d^{j}}{d \zeta^{j}} \nu(\zeta), \quad j=\beta .
\end{array}\right.
$$

\section{Idea of NDM}

The general fractional-order PDE is given as

$$
D^{\beta} \mu(\phi, \tau)+L \mu(\phi, \tau)+N \mu(\phi, \tau)=q(\phi, \tau), \quad \phi, \tau \geq 0,1<\beta \leq 1,
$$

In Equation (9), we represent the linear part of the equation with $L$ and the nonlinear part with $N$, and $D^{\beta}=\partial^{\beta} / \partial \tau^{\beta}$ denotes the Caputo fractional derivatives.

With initial condition,

$$
\mu(\phi, 0)=k(\phi)
$$
(9)

We have applied the Elzaki transformation to Equation

$$
\mathbb{E}\left[D^{\beta} \mu(\phi, \tau)\right]+\mathbb{E}[L \mu(\phi, \tau)+N \mu(\phi, \tau)]=\mathbb{E}[q(\phi, \tau)],
$$

and using Elzaki Transform's differentiation property, we 
get

$\frac{1}{s^{\beta}} \mathbb{E}[\mu(\phi, \tau)]-s^{2-\beta} \mu(\phi, 0)=\mathbb{E}[q(\phi, \tau)]-\mathbb{E}[L \mu(\phi, \tau)+N \mu(\phi, \tau)]$,

$\mathbb{E}[\mu(\phi, \tau)]=s^{2} \mu(\phi, 0)+s^{\beta} \mathbb{E}[q(\phi, \tau)]-s^{\beta} \mathbb{E}[L \mu(\phi, \tau)+N \mu(\phi, \tau)]$.

Now, $\mu(\phi, 0)=k(\phi)$.

$\mathbb{E}[\mu(\phi, \tau)]=s^{2} k(\phi)+s^{\beta} \mathbb{E}[q(\phi, \tau)]-s^{\beta} \mathbb{E}[L \mu(\phi, \tau)+N \mu(\phi, \tau)]$.

The following infinite series represent the EDM solution $\mu(\phi, \tau)$.

$$
\mu(\phi, \tau)=\sum_{j=0}^{\infty} \mu_{j}(\phi, \tau)
$$

and Adomian polynomials as

$$
\begin{gathered}
N \mu(\phi, \tau)=\sum_{j=0}^{\infty} A_{j}, \\
A_{j}=\frac{1}{j !}\left[\frac{d^{j}}{d \lambda^{j}}\left[N \sum_{j=0}^{\infty}\left(\lambda^{j} \mu_{j}\right)\right]\right]_{\lambda=0}, \quad j=0,1,2, \cdots .
\end{gathered}
$$

We get replacement Equation (14) and Equation (15) in Equation (13).

$\mathbb{E}\left[\sum_{j=0}^{\infty} \mu_{j}(\phi, \tau)\right]=s^{2} k(\phi)+s^{\beta} \mathbb{E}[q(\phi, \tau)]-s^{\beta} \mathbb{E}\left[L \sum_{j=0}^{\infty} \mu_{j}(\phi, \tau)+\sum_{j=0}^{\infty} A_{j}\right]$.

Applying the Elzaki transformation's linearity,

$$
\begin{array}{r}
\mathbb{E}\left[\mu_{0}(\phi, \tau)\right]=s^{2} k(\phi)+s^{\beta} \mathbb{E}[q(\phi, \tau)], \\
\mathbb{E}\left[\mu_{1}(\phi, \tau)\right]=-s^{\beta} \mathbb{E}\left[L \mu_{0}(\phi, \tau)+A_{0}\right] .
\end{array}
$$

We can generally write

$$
\mathbb{E}\left[\mu_{j+1}(\phi, \tau)\right]=-s^{\beta} \mathbb{E}\left[L \mu_{j}(\phi, \tau)+A_{j}\right], \quad j \geq 1
$$

Equation (18) and Equation (20) implement the inverse Elzaki transformation

$$
\begin{gathered}
\mu_{0}(\phi, \tau)=k(\phi)+\mathbb{E}^{-1}\left[s^{\beta} \mathbb{E}[q(\phi, \tau)]\right] \\
\mu_{j+1}(\phi, \tau)=-\mathbb{E}^{-1}\left[s^{\beta} \mathbb{E}\left[L \mu_{j}(\phi, \tau)+A_{j}\right]\right] .
\end{gathered}
$$

\section{Numerical Implementation}

4.1. Problem. Consider fractional-order one-dimensional parabolic equation:

$$
\frac{\partial^{\beta} \mu}{\partial \tau^{\beta}}+\left(\frac{1}{\phi}+\frac{\phi^{4}}{120}\right) \frac{\partial^{4} \mu}{\partial \phi^{4}}=0, \quad 1<\beta \leq 2, \tau \geq 0,
$$

with initial conditions

$$
\mu(\phi, 0)=0, \mu_{\tau}(\phi, 0)=1+\frac{\phi^{5}}{120},
$$

with boundary conditions

$$
\begin{gathered}
\mu\left(\frac{1}{2}, \tau\right)=\left(1+\frac{(1 / 2)^{5}}{120}\right) \sin (\tau), \mu(1, \tau)=\left(\frac{121}{120}\right) \sin (\tau), \\
\frac{\partial^{2} \mu}{\partial \phi^{2}}\left(\frac{1}{2}, \tau\right)=\frac{1}{6}\left(\frac{1}{2}\right)^{3} \sin (\tau), \frac{\partial^{2} \mu}{\partial \phi^{2}}(1, \tau)=\frac{1}{6} \sin (\tau) .
\end{gathered}
$$

The Elzaki transform of Equation (22):

$$
\frac{1}{s^{\beta}} \mu(\phi, s, u)-s^{2-\beta} \mu(\phi, 0)-s^{3-\beta} \mu_{\tau}(\phi, 0)=-\mathbb{E}\left[\left(\frac{1}{\phi}+\frac{\phi^{4}}{120}\right) \frac{\partial^{4} \mu}{\partial \phi^{4}}\right] .
$$

Simplify and replace Equation (23) condition.

$$
\mu(\phi, s, u)=s^{2}(0)+s^{3}\left(1+\frac{\phi^{5}}{120}\right)-\mathbb{E}\left[\left(\frac{1}{\phi}+\frac{\phi^{4}}{120}\right) \frac{\partial^{4} \mu}{\partial \phi^{4}}\right] .
$$

Use of inverse Elzaki transformation

$$
\mu(\phi, \tau)=\mathbb{E}^{-1}\left[s^{3}\left(1+\frac{\phi^{5}}{120}\right)-s^{\beta} \mathbb{E}\left[\left(\frac{1}{\phi}+\frac{\phi^{4}}{120}\right) \frac{\partial^{4} \mu}{\partial \phi^{4}}\right]\right],
$$

$$
\mu(\phi, \tau)=\left(1+\frac{\phi^{5}}{120}\right) \tau-\mathbb{E}^{-1}\left[s^{\beta} \mathbb{E}\left[\left(\frac{1}{\phi}+\frac{\phi^{4}}{120}\right) \frac{\partial^{4} \mu}{\partial \phi^{4}}\right]\right] .
$$

Equation (28) correction function is provided by

$$
\sum_{\ell=0}^{\infty} \mu_{\ell+1}(\phi, \tau)=\left(1+\frac{\phi^{5}}{120}\right) \tau-\mathbb{E}^{-1}\left[s^{\beta} \mathbb{E}\left[\left(\frac{1}{\phi}+\frac{\phi^{4}}{120}\right) \sum_{\ell=0}^{\infty} \frac{\partial^{4} \mu_{\ell}}{\partial \phi^{4}}\right]\right],
$$

the first term

$$
\mu_{0}(\phi, \tau)=\left(1+\frac{\phi^{5}}{120}\right) \tau,
$$


then we got

$$
\mu_{\ell+1}(\phi, \tau)=-\mathbb{E}^{-1}\left[s^{\beta} \mathbb{E}\left[\left(\frac{1}{\phi}+\frac{\phi^{4}}{120}\right) \sum_{\ell=0}^{\infty} \frac{\partial^{4} \mu_{\ell}}{\partial \phi^{4}}\right]\right]
$$

for $j=0$,

$$
\begin{gathered}
\mu_{1}(\phi, \tau)=-\mathbb{E}^{-1}\left[s^{\beta} \mathbb{E}\left[\left(\frac{1}{\phi}+\frac{\phi^{4}}{120}\right) \frac{\partial^{4} \mu_{0}}{\partial \phi^{4}}\right]\right], \\
\mu_{1}(\phi, \tau)=-\mathbb{E}^{-1}\left[\frac{\left(1+\left(\phi^{5} / 120\right)\right) u^{\beta}}{s^{\beta+2}}\right]=-\left(1+\frac{\phi^{5}}{120}\right) \frac{\tau^{\beta+1}}{\Gamma(\beta+2)} .
\end{gathered}
$$

The following terms are

$$
\begin{aligned}
& \mu_{2}(\phi, \tau)=-\mathbb{E}^{-1}\left[s^{\beta} \mathbb{E}\left[\left(\frac{1}{\phi}+\frac{\phi^{4}}{120}\right) \frac{\partial^{4} \mu_{1}}{\partial \phi^{4}}\right]\right]=\left(1+\frac{\phi^{5}}{120}\right) \frac{\tau^{2 \beta+1}}{\Gamma(2 \beta+2)}, \\
& \mu_{3}(\phi, \tau)=-\mathbb{E}^{-1}\left[s^{\beta} \mathbb{E}\left[\left(\frac{1}{\phi}+\frac{\phi^{4}}{120}\right) \frac{\partial^{4} \mu_{2}}{\partial \phi^{4}}\right]\right]=-\left(1+\frac{\phi^{5}}{120}\right) \frac{\tau^{3 \beta+1}}{\Gamma(3 \beta+2)},
\end{aligned}
$$

The series form of problem (1) such as:

$$
\begin{gathered}
\mu(\phi, \tau)=\mu_{0}(\phi, \tau)+\mu_{1}(\phi, \tau)+\mu_{2}(\phi, \tau)+\mu_{3}(\phi, \tau)+\mu_{4}(\phi, \tau) \cdots \\
\mu(\phi, \tau)=\left(1+\frac{\phi^{5}}{120}\right)\left\{\tau-\frac{\tau^{\beta+1}}{\Gamma(\beta+2)}+\frac{\tau^{2 \beta+1}}{\Gamma(2 \beta+2)}-\frac{\tau^{3 \beta+1}}{\Gamma(3 \beta+2)}+\frac{\tau^{4 \beta+1}}{\Gamma(4 \beta+2)} \cdots\right\},
\end{gathered}
$$

when $\beta=2$, then integer EDM solution is

$$
\mu(\phi, \tau)=\left(1+\frac{\phi^{5}}{120}\right)\left\{\tau-\frac{\tau^{3}}{3 !}+\frac{\tau^{5}}{5 !}-\frac{\tau^{7}}{7 !}+\frac{\tau^{9}}{9 !} \cdots\right\}
$$

The exact result is given as

$$
\mu(\phi, \tau)=\left(1+\frac{\phi^{5}}{120}\right) \sin (\tau)
$$

In Figure 1, the exact and the EDM solutions of problem 1 at $\beta=1$ are shown by subgraphs, respectively. From the given figure, it can be seen that both the EDM and exact results are in close contact with each other. In Figure 2, the EDM solutions of problem 1 are investigated at different fractional order $\beta=0.8$ and 0.6 . It is analyzed that timefractional problem results are convergent to an integerorder effect as time-fractional analysis to integer order.
4.2. Problem. Consider fractional-order two-dimensional parabolic equation:

$\frac{\partial^{\beta} \mu}{\partial \tau^{\beta}}+2\left(\frac{1}{\phi^{2}}+\frac{\phi^{4}}{6 !}\right) \frac{\partial^{4} \mu}{\partial \phi^{4}}+2\left(\frac{1}{\varphi^{2}}+\frac{\varphi^{4}}{6 !}\right) \frac{\partial^{4} \mu}{\partial \varphi^{4}}=0, \quad 1<\beta \leq 2, \tau \geq 0$,

with initial conditions

$$
\mu(\phi, \varphi, 0)=0, \mu_{\tau}(\phi, \varphi, 0)=2+\frac{\phi^{6}}{6 !}+\frac{\varphi^{6}}{6 !}
$$

with boundary conditions

$$
\begin{gathered}
\mu\left(\frac{1}{2}, \varphi, \tau\right)=\left(2+\frac{(1 / 2)^{6}}{6 !}+\frac{\varphi^{6}}{6 !}\right) \sin (\tau), \mu\left(\frac{1}{2}, \varphi, \tau\right)=\left(2+\frac{(1)^{6}}{6 !}+\frac{\varphi^{6}}{6 !}\right) \sin (\tau), \\
\mu_{\phi \phi}\left(\frac{1}{2}, \varphi, \tau\right)=\left(\frac{(1 / 2)^{4}}{4 !}\right) \sin (\tau), \mu_{\phi \phi}\left(\frac{1}{2}, \varphi, \tau\right)=\frac{1}{24} \sin (\tau), \\
\mu_{\varphi \varphi}\left(\phi, \frac{1}{2}, \tau\right)=\frac{(1 / 2)^{4}}{4 !} \sin (\tau), \mu_{\varphi \varphi}\left(\phi, \frac{1}{2}, \tau\right)=\frac{1}{24} \sin (\tau) .
\end{gathered}
$$

In the Elzaki transformation of Equation (37), we get

$$
\begin{aligned}
\frac{1}{s^{\beta}} & \mu(\phi, \varphi, s, u)-s^{2-\beta} \mu(\phi, 0)-s^{3-\beta} \mu_{\tau}(\phi, 0) \\
= & -\mathbb{E}\left[2\left(\frac{1}{\phi^{2}}+\frac{\phi^{4}}{6 !}\right) \frac{\partial^{4} \mu}{\partial \phi^{4}}+2\left(\frac{1}{\varphi^{2}}+\frac{\varphi^{4}}{6 !}\right) \frac{\partial^{4} \mu}{\partial \varphi^{4}}\right],
\end{aligned}
$$

Simplify and replace Equation (38) condition.

$$
\begin{aligned}
\mu(\phi, \varphi, s, u)= & s^{2}(0)+s^{3}\left(2+\frac{\phi^{6}}{6 !}+\frac{\varphi^{6}}{6 !}\right) \\
& -s^{\beta} \mathbb{E}\left[2\left(\frac{1}{\phi^{2}}+\frac{\phi^{4}}{6 !}\right) \frac{\partial^{4} \mu}{\partial \phi^{4}}+2\left(\frac{1}{\varphi^{2}}+\frac{\varphi^{4}}{6 !}\right) \frac{\partial^{4} \mu}{\partial \varphi^{4}}\right]
\end{aligned}
$$

using inverse Elzaki transformation

$$
\mu(\phi, \varphi, \tau)=\mathbb{E}^{-1}\left[s^{3}\left(2+\frac{\phi^{6}}{6 !}+\frac{\varphi^{6}}{6 !}\right)-s^{\beta} \mathbb{E}\left\{2\left(\frac{1}{\phi^{2}}+\frac{\phi^{4}}{6 !}\right) \frac{\partial^{4} \mu}{\partial \phi^{4}}+2\left(\frac{1}{\varphi^{2}}+\frac{\varphi^{4}}{6 !}\right) \frac{\partial^{4} \mu}{\partial \varphi^{4}}\right\}\right],
$$

$$
\begin{aligned}
\mu(\phi, \varphi, \tau)= & \left(2+\frac{\phi^{6}}{6 !}+\frac{\varphi^{6}}{6 !}\right) \tau-\mathbb{E}^{-1} \\
& \cdot\left[s^{\beta} \mathbb{E}\left\{2\left(\frac{1}{\phi^{2}}+\frac{\phi^{4}}{6 !}\right) \frac{\partial^{4} \mu}{\partial \phi^{4}}+2\left(\frac{1}{\varphi^{2}}+\frac{\varphi^{4}}{6 !}\right) \frac{\partial^{4} \mu}{\partial \varphi^{4}}\right\}\right],
\end{aligned}
$$



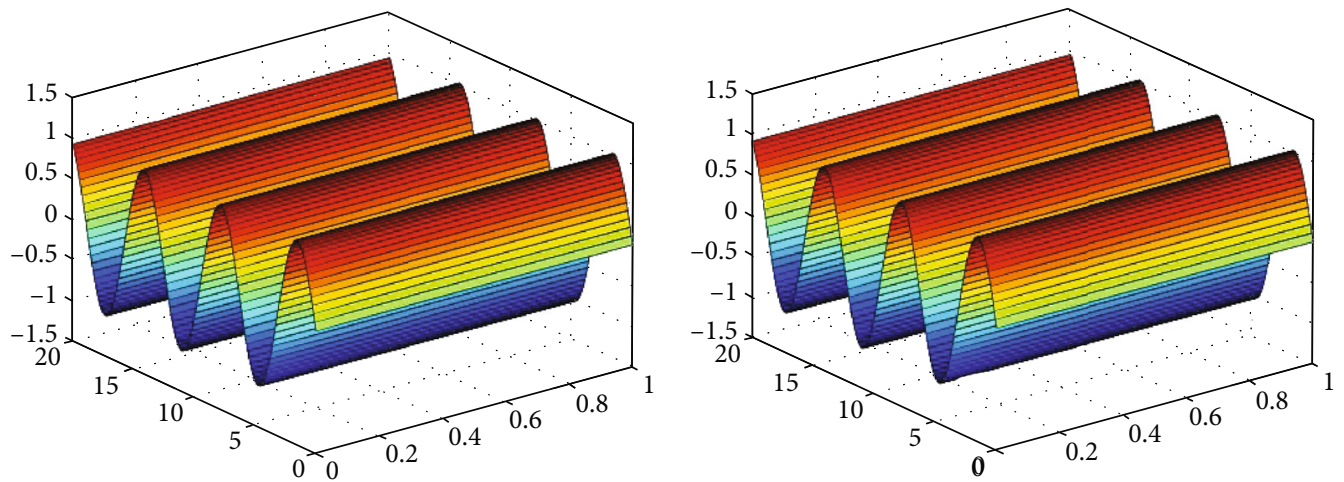

FIgURE 1: The graphs of exact and EDM result for $\beta=2$ of problem 1.
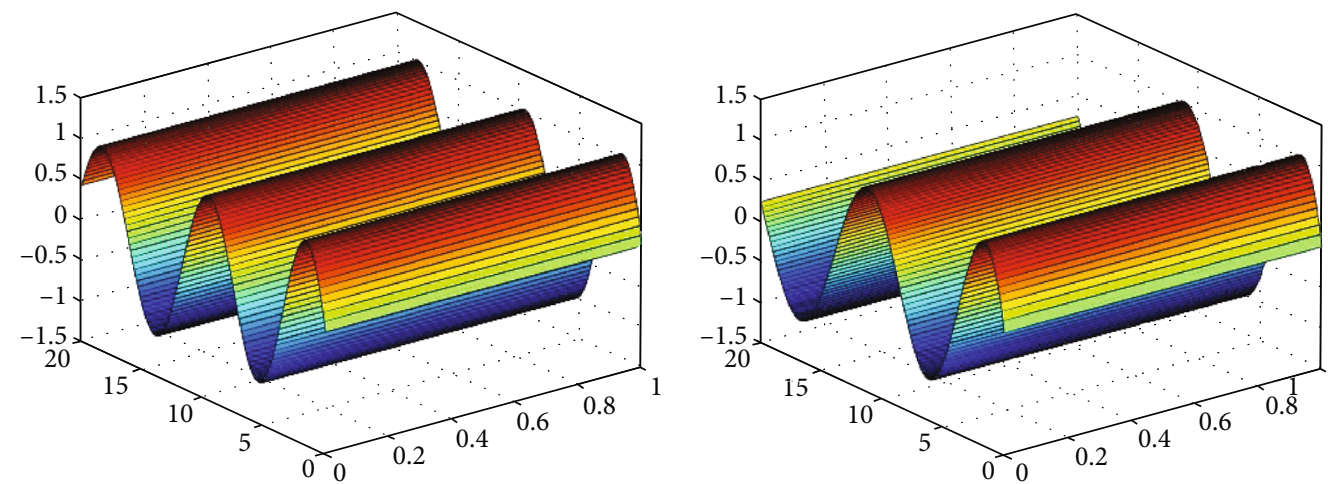

Figure 2: The fractional-order graphs of $\beta=0.8$ and 0.6 of problem 1 .

Equation (43) correction function is provided by

$$
\begin{aligned}
\sum_{\ell=0}^{\infty} \mu_{\ell+1}(\phi, \varphi, \tau)= & \left(2+\frac{\phi^{6}}{6 !}+\frac{\varphi^{6}}{6 !}\right) \tau-\mathbb{E}^{-1} \\
& \cdot\left[s^{\beta} \mathbb{E}\left\{2\left(\frac{1}{\phi^{2}}+\frac{\phi^{4}}{6 !}\right) \sum_{\ell=0}^{\infty} \frac{\partial^{4} \mu_{\ell}}{\partial \phi^{4}}+2\left(\frac{1}{\varphi^{2}}+\frac{\varphi^{4}}{6 !}\right) \sum_{\ell=0}^{\infty} \frac{\partial^{4} \mu_{\ell}}{\partial \varphi^{4}}\right\}\right],
\end{aligned}
$$

the first term

$$
\mu_{0}(\phi, \varphi, \tau)=\left(2+\frac{\phi^{6}}{6 !}+\frac{\varphi^{6}}{6 !}\right) \tau
$$

then we get

$\mu_{\ell+1}(\phi, \varphi, \tau)=-\mathbb{E}^{-1}\left[s^{\beta} \mathbb{E}\left\{2\left(\frac{1}{\phi^{2}}+\frac{\phi^{4}}{6 !}\right) \sum_{\ell=0}^{\infty} \frac{\partial^{4} \mu_{\ell}}{\partial \phi^{4}}+2\left(\frac{1}{\varphi^{2}}+\frac{\varphi^{4}}{6 !}\right) \sum_{\ell=0}^{\infty} \frac{\partial^{4} \mu_{\ell}}{\partial \varphi^{4}}\right\}\right]$,

for $j=0$,

$$
\begin{aligned}
& \mu_{1}(\phi, \varphi, \tau)=-\mathbb{E}^{-1}\left[s^{\beta} \mathbb{E}\left\{2\left(\frac{1}{\phi^{2}}+\frac{\phi^{4}}{6 !}\right) \frac{\partial^{4} \mu_{0}}{\partial \phi^{4}}+2\left(\frac{1}{\varphi^{2}}+\frac{\varphi^{4}}{6 !}\right) \frac{\partial^{4} \mu_{0}}{\partial \varphi^{4}}\right\}\right], \\
& \mu_{1}(\phi, \tau)=-\mathbb{E}^{-1}\left[\left(2+\frac{\phi^{6}}{6 !}+\frac{\varphi^{6}}{6 !}\right) \frac{u^{\beta}}{s^{\beta+2}}\right]=-\left(2+\frac{\phi^{6}}{6 !}+\frac{\varphi^{6}}{6 !}\right) \frac{\tau^{\beta+1}}{\Gamma(\beta+2)} .
\end{aligned}
$$

The following terms are

$$
\begin{gathered}
\mu_{2}(\phi, \varphi, \tau)=-\mathbb{E}^{-1}\left[s^{\beta} \mathbb{E}\left\{2\left(\frac{1}{\phi^{2}}+\frac{\phi^{4}}{6 !}\right) \frac{\partial^{4} \mu_{1}}{\partial \phi^{4}}+2\left(\frac{1}{\varphi^{2}}+\frac{\varphi^{4}}{6 !}\right) \frac{\partial^{4} \mu_{1}}{\partial \varphi^{4}}\right\}\right], \\
\mu_{2}(\phi, \varphi, \tau)=\left(2+\frac{\phi^{6}}{6 !}+\frac{\varphi^{6}}{6 !}\right) \frac{\tau^{2 \beta+1}}{\Gamma(2 \beta+2)}, \\
\mu_{3}(\phi, \varphi, \tau)=-\mathbb{E}^{-1}\left[s^{\beta} \mathbb{E}\left\{2\left(\frac{1}{\phi^{2}}+\frac{\phi^{4}}{6 !}\right) \frac{\partial^{4} \mu_{2}}{\partial \phi^{4}}+2\left(\frac{1}{\varphi^{2}}+\frac{\varphi^{4}}{6 !}\right) \frac{\partial^{4} \mu_{2}}{\partial \varphi^{4}}\right\}\right], \\
\mu_{3}(\phi, \varphi, \tau)=-\left(2+\frac{\phi^{6}}{6 !}+\frac{\varphi^{6}}{6 !}\right) \frac{\tau^{3 \beta+1}}{\Gamma(3 \beta+2)},
\end{gathered}
$$

In the series form of problem (2), we get

$$
\begin{aligned}
\mu(\phi, \varphi, \tau)= & \mu_{0}(\phi, \varphi, \tau)+\mu_{1}(\phi, \varphi, \tau)+\mu_{2}(\phi, \varphi, \tau) \\
& +\mu_{3}(\phi, \varphi, \tau)+\mu_{4}(\phi, \varphi, \tau) \cdots,
\end{aligned}
$$

$$
\begin{aligned}
\mu(\phi, \varphi, \tau)= & \left(2+\frac{\phi^{6}}{6 !}+\frac{\varphi^{6}}{6 !}\right)\left\{\tau-\frac{\tau^{\beta+1}}{\Gamma(\beta+2)}+\frac{\tau^{2 \beta+1}}{\Gamma(2 \beta+2)}\right. \\
& \left.-\frac{\tau^{3 \beta+1}}{\Gamma(3 \beta+2)}+\frac{\tau^{4 \beta+1}}{\Gamma(4 \beta+2)} \cdots\right\}
\end{aligned}
$$


Then, $\beta=2$, the integer EDM result as

$\mu(\phi, \varphi, \tau)=\left(2+\frac{\phi^{6}}{6 !}+\frac{\varphi^{6}}{6 !}\right)\left\{\tau-\frac{\tau^{3}}{3 !}+\frac{\tau^{5}}{5 !}-\frac{\tau^{7}}{7 !}+\frac{\tau^{9}}{9 !} \cdots\right\}$

The exact solution is

$$
\mu(\phi, \varphi, \tau)=\left(2+\frac{\phi^{6}}{6 !}+\frac{\varphi^{6}}{6 !}\right) \sin (\tau)
$$

In Figure 3, the exact and the EDM solutions of problem 2 at $\beta=1$ are shown by subgraphs, respectively. From the given figure, it can be seen that both the EDM and exact results are in close contact with each other. In Figure 4, the EDM solutions of problem 2 are investigated at different fractional order $\beta=0.8$ and 0.6 . It is analyzed that timefractional problem results are convergent to an integerorder effect as time-fractional analysis to integer order.

4.3. Problem. Consider fractional-order three-dimensional parabolic equation:

$\frac{\partial^{\beta} \mu}{\partial \tau^{\beta}}+2\left(\frac{\varphi+\psi}{2 \cos \phi}-1\right) \frac{\partial^{4} \mu}{\partial \phi^{4}}+2\left(\frac{\phi+\psi}{2 \cos \varphi}-1\right) \frac{\partial^{4} \mu}{\partial \varphi^{4}}+2\left(\frac{\varphi+\phi}{2 \cos \psi}-1\right) \frac{\partial^{4} \mu}{\partial \psi^{4}}=0$,

$$
1<\beta \leq 2, \tau \geq 0
$$

with initial conditions

$$
\mu(\phi, \varphi, \psi, 0)=\phi+\varphi+\psi-(\cos (\phi)+\cos (\varphi)+\cos (\psi)),
$$

$\mu_{\tau}(\phi, \varphi, \psi, 0)=(\cos (\phi)+\cos (\varphi)+\cos (\psi))-(\phi+\varphi+\psi)$,

with boundary conditions

$$
\begin{gathered}
\mu(0, \varphi, \psi, \tau)=(-1+\varphi+\psi-\cos (\varphi)-\cos (\psi)) e^{-\tau}, \\
\mu\left(\frac{\pi}{3}, \varphi, \psi, \tau\right)=\left(\frac{2 \pi-3}{6}+\varphi+\psi-\cos (\varphi)-\cos (\psi)\right) e^{-\tau}, \\
\mu(\phi, 0, \psi, \tau)=(-1+\phi+\psi-\cos (\phi)-\cos (\psi)) e^{-\tau}, \\
\mu\left(\phi, \frac{\pi}{3}, \psi, \tau\right)=\left(\frac{2 \pi-3}{6}+\phi+\psi-\cos (\phi)-\cos (\psi)\right) e^{-\tau}, \\
\mu(\phi, \varphi, 0, \tau)=(-1+\phi+\varphi-\cos (\phi)-\cos (\varphi)) e^{-\tau}, \\
\mu\left(\phi, \varphi, \frac{\pi}{3}, \tau\right)=\left(\frac{2 \pi-3}{6}+\phi+\varphi-\cos (\phi)-\cos (\varphi)\right) e^{-\tau}, \\
\mu_{\phi}(0, \varphi, \psi, \tau)=\mu_{\varphi}(\phi, 0, \psi, \tau)=\mu_{\psi}(\phi, \varphi, 0, \tau)=e^{-\tau}, \\
\mu_{\phi}\left(\frac{\pi}{3}, \varphi, \psi, \tau\right)=\mu_{\varphi}\left(\phi, \frac{\pi}{3}, \psi, \tau\right)=\mu_{\psi}\left(\phi, \varphi, \frac{\pi}{3}, \tau\right)=\left(\frac{\sqrt{3}+2}{2}\right) e^{-\tau} .
\end{gathered}
$$

In the Elzaki transformation of Equation (52), we get

$$
\begin{aligned}
& \frac{1}{s^{\beta}} \mu(\phi, \varphi, \psi, s, u)-s^{2-\beta} \mu(\phi, \varphi, \psi, 0)-s^{3-\beta} \mu_{\tau}(\phi, \varphi, \psi, 0) \\
& =-\mathbb{E}\left[2\left(\frac{\varphi+\psi}{2 \cos \phi}-1\right) \frac{\partial^{4} \mu}{\partial \phi^{4}}+2\left(\frac{\phi+\psi}{2 \cos \varphi}-1\right) \frac{\partial^{4} \mu}{\partial \varphi^{4}}\right. \\
& \left.\quad+2\left(\frac{\varphi+\phi}{2 \cos \psi}-1\right) \frac{\partial^{4} \mu}{\partial \psi^{4}}\right],
\end{aligned}
$$

Simplify and replace Equation (54) condition.

$$
\begin{aligned}
\mu(\phi, \varphi, \psi, s, u)= & s^{2}\{\phi+\varphi+\psi-(\cos (\phi)+\cos (\varphi)+\cos (\psi))\} \\
& +s^{3}\{(\cos (\phi)+\cos (\varphi)+\cos (\psi))-(\phi+\varphi+\psi)\} \\
& -s^{\beta} \mathbb{E}\left[2\left(\frac{\varphi+\psi}{2 \cos \phi}-1\right) \frac{\partial^{4} \mu}{\partial \phi^{4}}+2\left(\frac{\phi+\psi}{2 \cos \varphi}-1\right) \frac{\partial^{4} \mu}{\partial \varphi^{4}}\right. \\
& \left.+2\left(\frac{\varphi+\phi}{2 \cos \psi}-1\right) \frac{\partial^{4} \mu}{\partial \psi^{4}}\right]
\end{aligned}
$$

using the inverse Elzaki transform

$$
\begin{aligned}
\mu(\phi, \varphi, \psi, \tau)= & \mathbb{E}^{-1}\left[s^{2}\{\phi+\varphi+\psi-(\cos (\phi)+\cos (\varphi)+\cos (\psi))\}\right. \\
& \left.+s^{3}\{(\cos (\phi)+\cos (\varphi)+\cos (\psi))-(\phi+\varphi+\psi)\}\right] \\
& -\mathbb{E}^{-1}\left[s ^ { \beta } \mathbb { E } \left[2\left(\frac{\varphi+\psi}{2 \cos \phi}-1\right) \frac{\partial^{4} \mu}{\partial \phi^{4}}+2\left(\frac{\phi+\psi}{2 \cos \varphi}-1\right) \frac{\partial^{4} \mu}{\partial \varphi^{4}}\right.\right. \\
& \left.\left.+2\left(\frac{\varphi+\phi}{2 \cos \psi}-1\right) \frac{\partial^{4} \mu}{\partial \psi^{4}}\right]\right]
\end{aligned}
$$

$$
\begin{aligned}
\mu(\phi, \varphi, \psi, \tau)= & \{\phi+\varphi+\psi-(\cos (\phi)+\cos (\varphi)+\cos (\psi))\}(1-\tau) \\
& -\mathbb{E}^{-1}\left[s ^ { \beta } \mathbb { E } \left[2\left(\frac{\varphi+\psi}{2 \cos \phi}-1\right) \frac{\partial^{4} \mu}{\partial \phi^{4}}+2\left(\frac{\phi+\psi}{2 \cos \varphi}-1\right) \frac{\partial^{4} \mu}{\partial \varphi^{4}}\right.\right. \\
& \left.\left.+2\left(\frac{\varphi+\phi}{2 \cos \psi}-1\right) \frac{\partial^{4} \mu}{\partial \psi^{4}}\right]\right] .
\end{aligned}
$$

Equation (59) correction function is provided by

$$
\begin{aligned}
\sum_{\ell=0}^{\infty} \mu_{\ell+1}(\phi, \varphi, \tau)= & \{\phi+\varphi+\psi-(\cos (\phi)+\cos (\varphi)+\cos (\psi))\}(1-\tau) \\
& -\mathbb{E}^{-1}\left[s ^ { \beta } \mathbb { E } \left[2\left(\frac{\varphi+\psi}{2 \cos \phi}-1\right) \sum_{\ell=0}^{\infty} \frac{\partial^{4} \mu_{\ell}}{\partial \phi^{4}}+2\left(\frac{\phi+\psi}{2 \cos \varphi}-1\right) \sum_{\ell=0}^{\infty} \frac{\partial^{4} \mu_{\ell}}{\partial \varphi^{4}}\right.\right. \\
& \left.\left.+2\left(\frac{\varphi+\phi}{2 \cos \psi}-1\right) \sum_{\ell=0}^{\infty} \frac{\partial^{4} \mu_{\ell}}{\partial \psi^{4}}\right]\right]
\end{aligned}
$$

the first term

$\mu_{0}(\phi, \varphi, \psi, \tau)=\{\phi+\varphi+\psi-(\cos (\phi)+\cos (\varphi)+\cos (\psi))\}(1-\tau)$, 

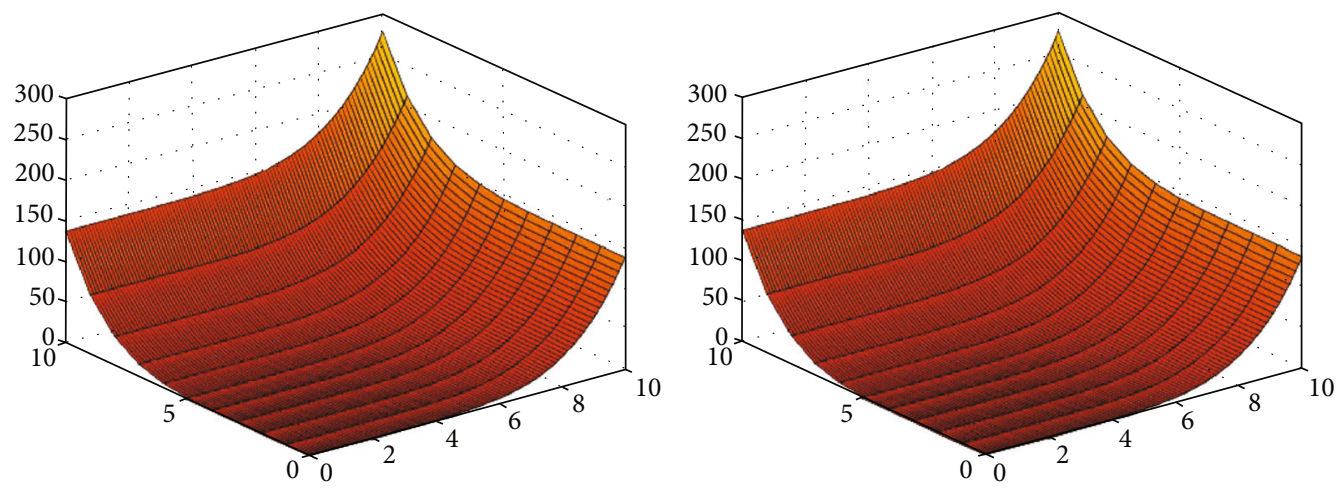

Figure 3: The exact and EDM solution for $\beta=2$ of problem 2.
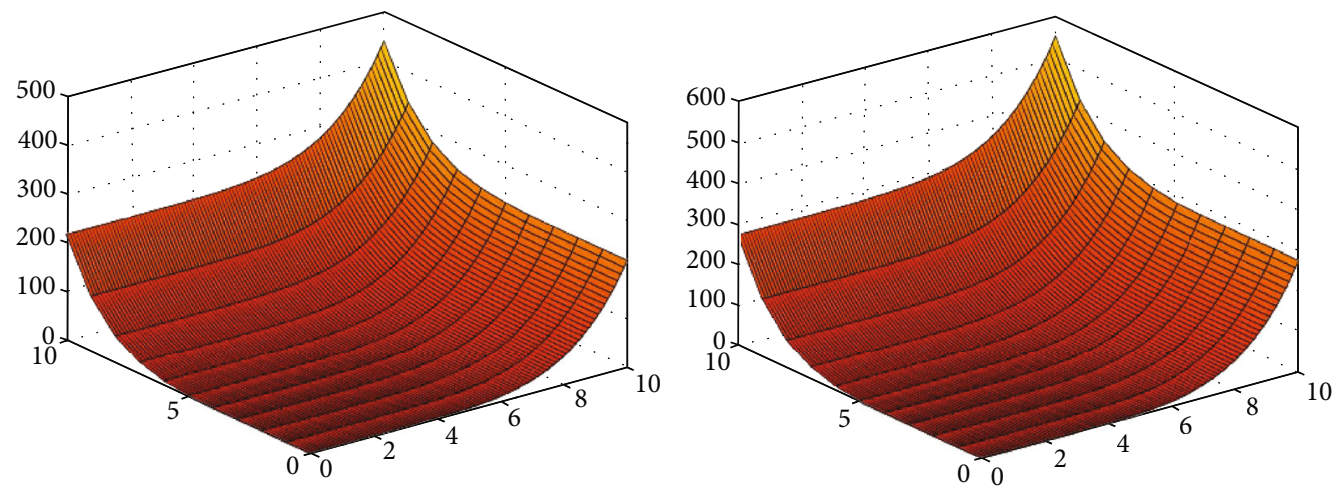

Figure 4: The fractional-order graphs of $\beta=0.8$ and 0.6 of problem 2.

then we get

$$
\begin{aligned}
\mu_{\ell+1}(\phi, \varphi, \psi, \tau)= & -\mathbb{E}^{-1}\left[s ^ { \beta } \mathbb { E } \left\{2\left(\frac{\varphi+\psi}{2 \cos \phi}-1\right) \sum_{\ell=0}^{\infty} \frac{\partial^{4} \mu_{\ell}}{\partial \phi^{4}}\right.\right. \\
& \left.\left.+2\left(\frac{\phi+\psi}{2 \cos \varphi}-1\right) \sum_{\ell=0}^{\infty} \frac{\partial^{4} \mu_{\ell}}{\partial \varphi^{4}}+2\left(\frac{\varphi+\phi}{2 \cos \psi}-1\right) \sum_{\ell=0}^{\infty} \frac{\partial^{4} \mu_{\ell}}{\partial \psi^{4}}\right\}\right],
\end{aligned}
$$

for $j=0$,

$$
\begin{aligned}
\mu_{1}(\phi, \varphi, \psi, \tau)= & -\mathbb{E}^{-1}\left[s ^ { \beta } \mathbb { E } \left\{2\left(\frac{\varphi+\psi}{2 \cos \phi}-1\right) \frac{\partial^{4} \mu_{0}}{\partial \phi^{4}}+2\left(\frac{\phi+\psi}{2 \cos \varphi}-1\right) \frac{\partial^{4} \mu_{0}}{\partial \varphi^{4}}\right.\right. \\
& \left.\left.+2\left(\frac{\varphi+\phi}{2 \cos \psi}-1\right) \frac{\partial^{4} \mu_{0}}{\partial \psi^{4}}\right\}\right],
\end{aligned}
$$

$\mu_{1}(\phi, \varphi, \psi, \tau)=\mathbb{E}^{-1}\left[\phi+\varphi+\psi-(\cos (\phi)+\cos (\varphi)+\cos (\psi))\left(\frac{u^{\beta}}{s^{\beta+1}}-\frac{u^{\beta}}{s^{\beta+2}}\right)\right]$,

$\mu_{1}(\phi, \varphi, \psi, \tau)=\{\phi+\varphi+\psi-(\cos (\phi)+\cos (\varphi)+\cos (\psi))\}\left(\frac{\tau^{\beta}}{\Gamma(\beta+1)}-\frac{\tau^{\beta+1}}{\Gamma(\beta+2)}\right)$.
The following terms are

$$
\begin{aligned}
\mu_{2}(\phi, \varphi, \psi, \tau)= & -\mathbb{E}^{-1}\left[s ^ { \beta } \mathbb { E } \left\{2\left(\frac{\varphi+\psi}{2 \cos \phi}-1\right) \frac{\partial^{4} \mu_{1}}{\partial \phi^{4}}\right.\right. \\
& \left.\left.+2\left(\frac{\phi+\psi}{2 \cos \varphi}-1\right) \frac{\partial^{4} \mu_{1}}{\partial \varphi^{4}}+2\left(\frac{\varphi+\phi}{2 \cos \psi}-1\right) \frac{\partial^{4} \mu_{1}}{\partial \psi^{4}}\right\}\right],
\end{aligned}
$$

$$
\begin{aligned}
& \mu_{2}(\phi, \varphi, \psi, \tau)=\{\phi+\varphi+\psi-(\cos (\phi)+\cos (\varphi)+\cos (\psi))\} \\
& \cdot\left(\frac{\tau^{2 \beta}}{\Gamma(2 \beta+1)}-\frac{\tau^{2 \beta+1}}{\Gamma(2 \beta+2)}\right), \\
& \mu_{3}(\phi, \varphi, \psi, \tau)=\mathbb{E}^{-1}\left[s ^ { \beta } \mathbb { E } \left\{2\left(\frac{\varphi+\psi}{2 \cos \phi}-1\right) \frac{\partial^{4} \mu_{2}}{\partial \phi^{4}}\right.\right. \\
&\left.\left.+2\left(\frac{\phi+\psi}{2 \cos \varphi}-1\right) \frac{\partial^{4} \mu_{2}}{\partial \varphi^{4}}+2\left(\frac{\varphi+\phi}{2 \cos \psi}-1\right) \frac{\partial^{4} \mu_{2}}{\partial \psi^{4}}\right\}\right],
\end{aligned}
$$

$\mu_{3}(\phi, \varphi, \psi, \tau)=\{\phi+\varphi+\psi-(\cos (\phi)+\cos (\varphi)+\cos (\psi))\}$

$$
\cdot\left(\frac{\tau^{3 \beta}}{\Gamma(3 \beta+1)}-\frac{\tau^{3 \beta+1}}{\Gamma(3 \beta+2)}\right) \text {, }
$$



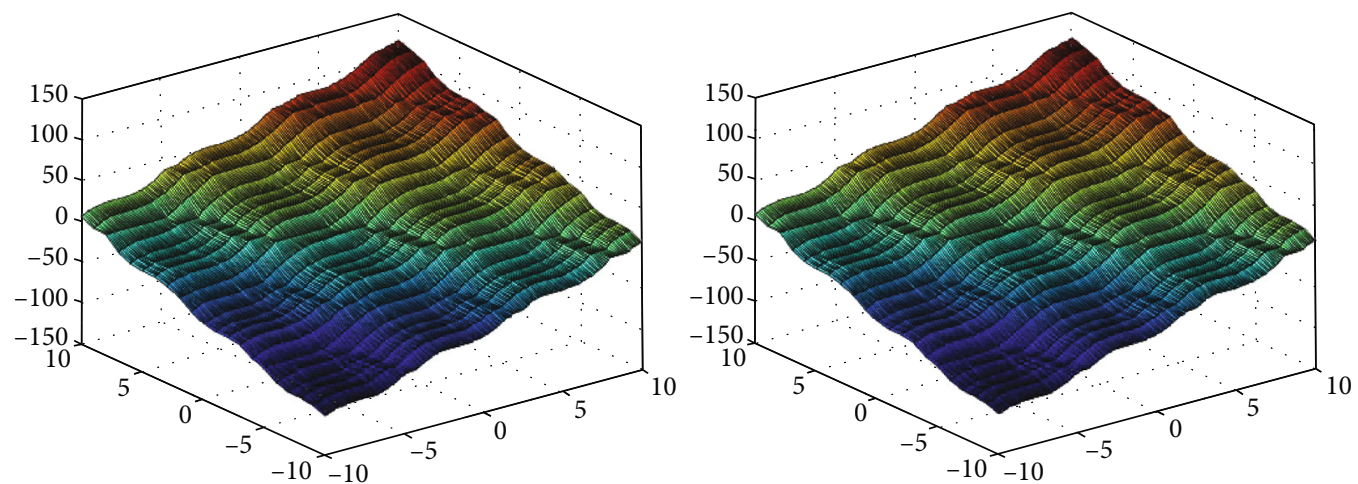

FIgURE 5: The exact and EDM solution for $\beta=2$ of problem 3.
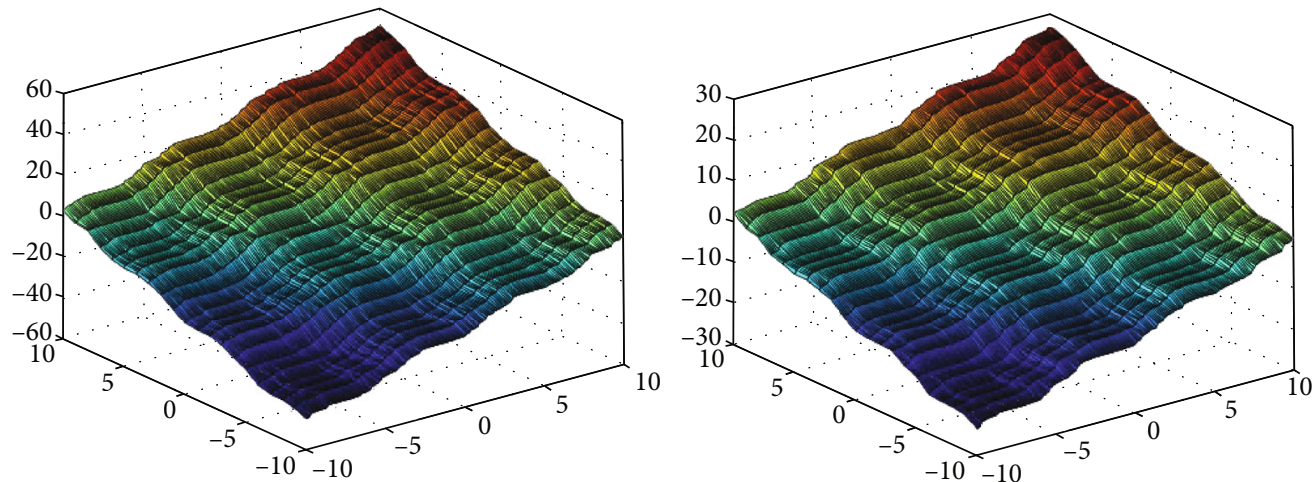

FIGURE 6: For fractional-order graphs of $\beta=0.8$ and 0.6 of problem 3 .

The series form of problem (3) such as

$$
\begin{aligned}
& \mu(\phi, \varphi, \psi, \tau)= \mu_{0}(\phi, \varphi, \psi, \tau)+\mu_{1}(\phi, \varphi, \psi, \tau)+\mu_{2}(\phi, \varphi, \psi, \tau) \\
&+\mu_{3}(\phi, \varphi, \psi, \tau)+\mu_{4}(\phi, \varphi, \psi, \tau) \cdots, \\
& \mu(\phi, \varphi, \psi, \tau)=\{\phi+\varphi+\psi-(\cos (\phi)+\cos (\varphi)+\cos (\psi))\} \\
& \cdot\left\{1-\tau+\frac{\tau^{\beta}}{\Gamma(\beta+1)}-\frac{\tau^{\beta+1}}{\Gamma(\beta+2)}+\frac{\tau^{2 \beta}}{\Gamma(2 \beta+1)}\right. \\
&\left.-\frac{\tau^{2 \beta+1}}{\Gamma(2 \beta+2)}+\frac{\tau^{3 \beta}}{\Gamma(3 \beta+1)}-\frac{\tau^{3 \beta+1}}{\Gamma(3 \beta+2)} \cdots\right\} .
\end{aligned}
$$

In the integer-order solution of EDM of Equation (52) at $\beta=2$, we get

$$
\begin{aligned}
\mu(\phi, \varphi, \psi, \tau)= & \{\phi+\varphi+\psi-(\cos (\phi)+\cos (\varphi)+\cos (\psi))\} \\
& \cdot\left\{1-\tau+\frac{\tau^{2}}{2 !}-\frac{\tau^{3}}{3 !}+\frac{\tau^{4}}{4 !}-\frac{\tau^{5}}{5 !}+\frac{\tau^{6}}{6 !}-\frac{7}{7 !} \cdots\right\} .
\end{aligned}
$$

The exact solution is given as

$$
\mu(\phi, \varphi, \psi, \tau)=(\phi+\varphi+\psi-(\cos (\phi)+\cos (\varphi)+\cos (\psi))) e^{-\tau} .
$$

In Figure 5, the exact and the EDM solutions of problem 3 at $\beta=1$ are shown by subgraphs, respectively. From the given figure, it can be seen that both the EDM and exact results are in close contact with each other. In Figure 6, the EDM solutions of problem 3 are investigated at different fractional order $\beta=0.8$ and 0.6 . It is analyzed that timefractional problem results are convergent to an integerorder effect as time-fractional analysis to integer order.

\section{Conclusion}

In the present article, an efficient analytical technique is used to solve fractional-order parabolic equations. The present method is the combination of two well-known methods, namely, Elzaki transform and Adomian decomposition method. The Elzaki transform is applied to the given problem, which makes it easier. After this, we implemented Adomian decomposition method and then inverse Elzaki transform to get closed form analytical solutions for the given problems. The proposed method required small number of calculation to attain closed form solutions and is therefore considered to be one of the best analytical method to solve fractional-order partial differential equations.

\section{Data Availability}

The numerical data used to support the findings of this study are included within the article. 


\section{Conflicts of Interest}

The authors declare that there are no conflicts of interest regarding the publication of this article.

\section{Acknowledgments}

One of the coauthors (A. M. Zidan) extends his appreciation to the Deanship of Scientific Research at King Khalid University, Abha, 61413, Saudi Arabia, for funding this work through a research group program under grant number R.G.P.-2/142/42. The authors would like to thank Deanship Scientific Research at the Umm Al-Qura University for supporting this research work under grant code 19-SCI-1-010041.

\section{References}

[1] A. Q. M. Khaliq and E. H. Twizell, "A family of second order methods for variable coefficient fourth order parabolic partial differential equations," International Journal of Computer Mathematics, vol. 23, no. 1, pp. 63-76, 1987.

[2] H. Jafari, J. G. Prasad, P. Goswami, and R. S. Dubey, "Solution of the local fractional generalized KDV equation using homotopy analysis method," Fractals, vol. 29, no. 5, article 2140014, 2021.

[3] D. J. Gorman, "Free vibration analysis of beams and shafts (book)," in Research supported by the National Research Council of Canada, p. 395, Wiley-Interscience, New York, 1975.

[4] C. Andrade and S. McKee, "High accuracy A.D.I. methods for fourth order parabolic equations with variable coefficients," Journal of Computational and Applied Mathematics, vol. 3, no. 1, pp. 11-14, 1977.

[5] R. S. Dubey, B. S. T. Alkahtani, and A. Atangana, "Analytical solution of space-time fractional Fokker-Planck equation by homotopy perturbation Sumudu transform method," Mathematical Problems in Engineering, vol. 2015, Article ID 780929, 7 pages, 2015.

[6] S. D. Conte, "A stable implicit finite difference approximation to a fourth order parabolic equation," Journal of the ACM, vol. 4, no. 1, pp. 18-23, 1957.

[7] V. Gill and R. S. Dubey, "New analytical method for KleinGordon equations arising in quantum field theory," European Journal of Advances in Engineering and Technology, vol. 5, no. 8, pp. 649-655, 2018.

[8] W. C. Royster and S. D. Conte, "Convergence of finite difference solutions to a solution of the equation of the vibrating rod," Proceedings of the American Mathematical Society, vol. 7, no. 4, pp. 742-749, 1956.

[9] V. B. L. Chaurasia and R. S. Dubey, "Analytical solution for the differential equation containing generalized fractional derivative operators and Mittag-Leffler-type function," International Scholarly Research Notices, vol. 2011, Article ID 682381, 9 pages, 2011.

[10] D. J. Evans, "A stable explicit method for the finite-difference solution of a fourth-order parabolic partial differential equation," The Computer Journal, vol. 8, no. 3, pp. 280-287, 1965.

[11] D. J. Evans and W. S. Yousif, "A note on solving the fourth order parabolic equation by the AGE method," International journal of computer mathematics, vol. 40, no. 1-2, pp. 93-97, 1991.
[12] A.-M. Wazwaz, "On the solution of the fourth order parabolic equation by the decomposition method," International Journal of Computer Mathematics, vol. 57, no. 3-4, pp. 213-217, 1995.

[13] M. A. Almuqrin, P. Goswami, S. Sharma, I. Khan, R. S. Dubey, and A. Khan, "Fractional model of Ebola virus in population of bats in frame of Atangana-Baleanu fractional derivative," Results in Physics, vol. 26, article 104295, 2021.

[14] A. Kilicman, R. Shokhanda, and P. Goswami, "On the solution of $(\mathrm{n}+1)$-dimensional fractional M-Burgers equation," Alexandria Engineering Journal, vol. 60, no. 1, pp. 1165-1172, 2021.

[15] I. Malyk, M. M. A. Shrahili, A. R. Shafay, P. Goswami, S. Sharma, and R. S. Dubey, "Analytical solution of nonlinear fractional Burger's equation in the framework of different fractional derivative operators," Results in Physics, vol. 19, article 103397, 2020.

[16] W. Liao, J. Zhu, and A. Q. M. Khaliq, "An efficient high-order algorithm for solving systems of reaction-diffusion equations," Numerical Methods for Partial Differential Equations, vol. 18, no. 3, pp. 340-354, 2002.

[17] M. K. Jain, S. R. K. Iyengar, and A. G. Lone, "Higher order difference formulas for a fourth order parabolic partial differential equation," International Journal for Numerical Methods in Engineering, vol. 10, no. 6, pp. 1357-1367, 1976.

[18] H. Caglar and N. Caglar, "Fifth-degree B-spline solution for a fourth-order parabolic partial differential equations," Applied Mathematics and Computation, vol. 201, no. 1-2, pp. 597603, 2008.

[19] A.-M. Wazwaz, "Analytic treatment for variable coefficient fourth-order parabolic partial differential equations," Applied Mathematics and Computation, vol. 123, no. 2, pp. 219-227, 2001.

[20] J. Rashidinia and R. Mohammadi, "Sextic spline solution of variable coefficient fourth-order parabolic equations," International Journal of Computer Mathematics, vol. 87, no. 15, pp. 3443-3454, 2010.

[21] T. Aziz, A. Khan, and J. Rashidinia, "Spline methods for the solution of fourth-order parabolic partial differential equations," Applied Mathematics and Computation, vol. 167, no. 1, pp. 153-166, 2005.

[22] J. Biazar and H. Ghazvini, "He's variational iteration method for fourth-order parabolic equations," Computers and Mathematics with Applications, vol. 54, no. 7-8, pp. 1047-1054, 2007.

[23] M. A. Noor, K. I. Noor, and S. T. Mohyud-Din, "Modified variational iteration technique for solving singular fourth-order parabolic partial differential equations," Nonlinear Analysis: Theory, Methods and Applications, vol. 71, no. 12, pp. e630-e640, 2009.

[24] M. Dehghan and J. Manafian, "The solution of the variable coefficients fourth-order parabolic partial differential equations by the homotopy perturbation method," Zeitschrift für Naturforschung A, vol. 64, no. 7-8, pp. 420-430, 2009.

[25] M. Nadeem, F. Li, and H. Ahmad, "Modified Laplace variational iteration method for solving fourth-order parabolic partial differential equation with variable coefficients," Computers and Mathematics with Applications, vol. 78, no. 6, pp. 20522062, 2019.

[26] G. Adomian, "Solving frontier problems of physics: the decomposition method; with a preface by Yves Cherruault," Fundamental Theories of Physics, vol. 60, 1994.

[27] T. M. Elzaki, "The new integral transform Elzaki transform," Global Journal of Pure and Applied Mathematics, vol. 7, no. 1, pp. 57-64, 2011. 
[28] T. M. Elzaki, "On the connections between Laplace and Elzaki transforms," Advances in Theoretical and Applied Mathematics, vol. 6, no. 1, pp. 1-11, 2011.

[29] T. M. Elzaki, "On the new integral transform "Elzaki transform" fundamental properties investigations and applications," Global Journal of Mathematical Sciences: Theory and Practical, vol. 4, no. 1, pp. 1-13, 2012.

[30] R. M. Jena and S. Chakraverty, "Solving time-fractional Navier-Stokes equations using homotopy perturbation Elzaki transform," SN Applied Sciences, vol. 1, no. 1, p. 16, 2019.

[31] A. K. H. Sedeeg, "A coupling Elzaki transform and homotopy perturbation method for solving nonlinear fractional heat-like equations," American Journal of Mathematical and Computer Modelling, vol. 1, no. 1, pp. 15-20, 2016.

[32] A. C. Loyinmi and T. K. Akinfe, "An algorithm for solving the Burgers-Huxley equation using the Elzaki transform," $S N$ Applied Sciences, vol. 2, no. 1, pp. 1-17, 2020. 